\title{
Wirkungsorientierte
}

\section{Steuerung im Dienste der \\ Organisationsentwicklung}

\section{Zu wissen, was man will und warum setzt einen Lernprozess voraus}

\section{MARIA LAURA BONO}

Maria Laura Bono hatte nach dem Studium der Wirtschaftswissenschaften in Graz und London mehrere Führungspositionen in NonProfit-Organisationen inne bevor sie 2007 in die Unternehmensberatung einstieg. Innerhalb der Integrated Consulting Group $\mathrm{GmbH}$ setzt sie sich insbesondere mit den Themen Steuerung sozialer Dienste, Strategiekonzeption und Strategieumsetzung sowie Evaluation auseinander. marialaura.bono@integratedconsulting.at

\author{
Soziale Dienste und Einrichtungen können \\ erfolgreich nur gesteuert werden, wenn in einem \\ umfassenden Diskurs von allen Beteiligten Klarheit \\ darüber geschaffen wurde, welche Wirkungen \\ die Organisation überhaupt erreichen will.
}

Spätestens seit der Jahrtausendwende ist Wirkungsorientierung zum Inbegriff eines professionellen Managements geworden. Zu einer Vorgabe, der man sich als Führungskraft nicht entziehen kann, und sei es auch nur, um vor den Stakeholdern die Legitimität zu bewahren.

Wirkungsorientierte Steuerung ist insbesondere für jene Organisationen zu einem Schlagwort geworden, die sich primär zu Sachzielen bekennen und so-

\section{Organisationales Lernen versus Legitimation}

Wirkungsorientierung kann verstanden werden als die stetige Ausrichtung des eigenen Handelns nach den erreichten Wirkungen und den geplanten Zielen. Wirkungsorientierung bewegt sich also in einem Kontinuum zwischen organisationalem Lernen und Legitimation (vgl. Abb. 1).

\section{"Bei Sparmaßnahmen geht es um finanzielle Kontrolle, nicht um ein fachliches Controlling"}

mit unter Druck stehen, definieren und dokumentieren zu müssen, was sie eigentlich erreichen wollen und inwieweit ihnen dies gelingt.

Es wäre jedoch verkürzt, lediglich die Legitimationsfunktion der Steuerung zu sehen. Wirkungsorientierung, gekonnt konzipiert und umgesetzt, kann und soll insbesondere der inhaltlichen Verbesserung der Organisation dienen.
Umso höher der externe Druck auf die Organisation ist, die eingesetzten Mittel zu rechtfertigen, desto stärker dient Steuerung dem Ziel, Legitimation zu schaffen und zu erhalten. Die Aufmerksamkeit gilt der Erarbeitung von Kennzahlen und dem Entwerfen von Berichtsbögen, während organisationales Lernen in den Hintergrund tritt. In einem solchen Fall wird Wirkungsorientierung meist als Top-down-Prozess 


\section{Externer Druck}

niedrig

hoch

\section{organisationales} Lernen

Abb. 1: Wirkungsorientierung pendelt zwischen organisationalem Lernen und Legitimation.

Quelle: Eigene Darstellung

praktiziert, was mit Machtverhältnissen und Kontrolle verbunden ist. Mitarbeiterinnen und Mitarbeiter sind häufig skeptisch, ob und was Steuerung, im Non-Profit-Bereich, der von so vielen Faktoren bestimmt wird, überhaupt bringen kann. Rückzug und Dienst nach Vorschrift sind häufige Folgen.

Findet der Diskurs über Aktivitäten und Wirkungen allerdings losgelöst von äußeren Vorgaben statt, kann sich ein fruchtbarer Dialog über Ziele und Mittel entwickeln. Im Mittelpunkt stehen dann die Zusammenführung von Know-how und die Erarbeitung einer gemeinsamen Basis, um die Vision der Organisation besser umzusetzen. Dies impliziert einen breit angelegten Prozess, der jedenfalls auch Buttomup-Elemente enthält, um Wissen und Erfahrungen auf allen Ebenen anzuzap- fen. Dadurch fühlen sich Mitarbeitende geschätzt und ernst genommen, was schließlich motivierend und leistungsfördernd wirkt.

\section{Erfolgreiches Steuern setzt Lernen voraus}

Meist wird bei der Entwicklung eines Steuerungssystems die größte Aufmerksamkeit der Konzeptarbeit geschenkt. In den Implementierungsprozess dagegen wird wenig investiert - fast so, als würde es genügen, Kennzahlen definiert zu haben, um einen Steuerungseffekt zu erzielen.

Entscheidend für die gelungene Implementierung eines Steuerungskreislaufes ist aber sicherzustellen, dass das Personal motiviert und bereit dazu ist, das entwickelte Konzept im Alltag an- zuwenden. Dies gelingt, wenn die Vorteile für den Einzelnen hervorgehoben werden: Sei es, dass die eigene Arbeit sichtbarer wird, dass Reibungsverluste minimiert werden können, weil die Abläufe transparenter werden oder, dass mit gegebenen Mitteln Ziele besser erreicht werden können. Das System von "promente kärnten " ist ein gutes Beispiel dafür, wie sich Steuerung und organisationales Lernen ergänzen und bestärken können (vgl. Kasten »Praxisbeispiel: Wie organisationales Lernen funktionieren kann«).

\section{Lernen baut auf Vertrauen auf}

Im Gegensatz zum bekannten Sprichwort gilt für Organisationen das umgekehrte Motto: »Kontrolle ist gut, Vertrauen ist besser." Es soll eine nachhaltige Lernkultur etabliert werden. Ein Klima des Vertrauens in die Fähigkeiten und in die Bereitschaft des Einzelnen zählt zu den nachgewiesenen Antriebsfaktoren für (Spitzen)Leistungen.

Vertrauen drückt sich in der Bereitschaft aus, das Risiko eines Misserfolgs in Kauf zu nehmen, in der Überzeugung, dass der Mitarbeitende dies nicht ausnützt, sondern konstruktiv damit umgeht. Kontrolle dagegen drückt Zweifel an den Fähigkeiten oder an der Integrität des Mitarbeitenden aus. Zugleich wird die Machtposition der Kontrollinstanz betont, die urteilen und möglicherweise verurteilen darf.

\begin{tabular}{|l|l|l|}
\hline Menschenbild & $\begin{array}{l}\text { Schwerpunkt der Organisationskultur: } \\
\text { Kontrolle }\end{array}$ & $\begin{array}{l}\text { Schwerpunkt der Organisationskultur: } \\
\text { Vertrauen }\end{array}$ \\
\hline Form der Ergebnisbegutachtung & $\begin{array}{l}\text { Der Mensch gilt als fehleranfällig und } \\
\text { in seiner Motivation schwankend. } \\
\text { Steuerungsgrößen sollen seine Funk- } \\
\text { tionen überprüfen, wie bei einer Ma- } \\
\text { schine. }\end{array}$ & $\begin{array}{l}\text { Der Mensch gilt als lernfähiges, } \\
\text { grundsätzlich leistungsbereites Indi- } \\
\text { viduum. Steuerungsgrößen sollen vor } \\
\text { allem Anhaltspunkte liefern, um sich } \\
\text { weiterentwickeln zu können. }\end{array}$ \\
\hline Fehlerkultur & $\begin{array}{l}\text { Das Ergebnis wird in kurzen Abstän- } \\
\text { den überprüft. }\end{array}$ & $\begin{array}{l}\text { Kontrollen werden abgelehnt; eine } \\
\text { Begutachtung des Ergebnisses dient } \\
\text { der Reflexion. }\end{array}$ \\
\hline $\begin{array}{l}\text { Fehler werden als Abweichung von } \\
\text { der Norm gesehen und sanktioniert. } \\
\text { Im Vordergrund steht die Suche nach } \\
\text { dem Schuldigen, nicht das Verstehen } \\
\text { und Optimieren der Vorgänge. }\end{array}$ & $\begin{array}{l}\text { Fehler werden als unvermeidbar gese- } \\
\text { hen. Wichtig ist die dahinter stehende } \\
\text { Intention und das, was aus Fehlern ge- } \\
\text { lernt wird. Das öffentliche Anschuldi- } \\
\text { gen von Einzelnen ist ausgeschlossen. }\end{array}$ \\
\hline
\end{tabular}

Abb. 2: Unterschiedliche Organisationskulturen haben folgenreiche Auswirkungen auf den Alltag in Diensten und Einrichtungen.

Quelle: Angepasst nach Heidbrink/Jenewein (2011), S. 69. 


\section{Praxisbeispiel: Wie organisationales Lernen funktionieren kann}

Die österreichische Non-Profit-Organisation "pro mente kärnten« ist ein Gesundheitsunternehmen und Mitglied im Dachverband pro mente austria. Die Organisation stellt Menschen mit psychischen, mentalen und sozialen Problemen alltagsorientierte Hilfen und Arbeitsplätze zur Verfügung, um ganzheitlich Gesundheit zu fördern, zu erhalten oder wiederherzustellen. Das Ziel ist ein hohes Maß an Gesundheit, Autonomie, Lebensqualität und Inklusion sowie Sensibilisierung und Akzeptanz der Gesellschaft. pro mente kärnten beschäftigt in Betreuungs- und Arbeitsprojekten mit einem Jahresumsatz von circa 14 Millionen Euro etwa 300 Mitarbeiterinnen und Mitarbeiter. Die Organisation deckt damit einen wesentlichen Teil der außerklinischen sozialpsychiatrischen Versorgung in Kärnten $a b$. Die Finanzierungsstruktur des Leistungsportfolios basiert zu 75 Prozent auf öffentlicher Finanzierung und zu 25 Prozent aus Eigenerwirtschaftung der Beschäftigungsprojekte.

Der Träger hatte im Zeitraum zwischen 1995 und 2005 aufgrund massiver Angebotsdiversifizierung eine Phase starken betrieblichen Wachstums zu bewältigen. Dieses generierte sich aus der prägenden Rolle der obersten Leitung unter Prim. Dr. Platz im Psychiatriediskurs der 199oer Jah$r$, in dem die Leitungsebene eine weitgehende inhaltliche Alleinstellung unter den dienstleistenden Diskurspartnern innehatte. Damit gingen allerdings Diskurserfolge meist auch in die operative Umsetzungsverantwortung des Trägers über, um diese für das Handlungsfeld der (sozial-) psychiatrischen Versorgung auch tatsächlich realisiert zu wissen.

Nicht nur das Angebot wurde erweitert, auch die Strukturen erfuhren eine entsprechende Anpassung. Da die Erweiterungsimpulse wesentlich aus der Teilnahme am sach- und landespolitischen Handlungsfelddiskurs entstanden, der naturgemäß weitgehend von den Führungskräften getragen wurde, entstand allerdings un- vermeidbar eine Top-down-Orientierung der Organisationsentwicklung, die in ihrem Komplexititätszuwachs das strategische Management vor organisationale Heraus forderungen stellte und den Legitimationsdruck steigerte.

Dem wurde u. a. ab dem Jahre 2002 durch die Implementation des strategischen Planungs- und Steuerungsinstrumentes der Balanced Score Card zu entsprechen gesucht. Im Wesentlichen wurden im ersten umgesetzten Modell quantitative Aspekte in hoher Komplexität operationalisiert.

Diese Komplexität zeitigte in der Folge Handhabungsschwierigkeiten des Instrumentes. Ebenso zeigte sich mangelnde Anschlussfähigkeit der Kennzahlen in der Wahrnehmung der Mitarbeiterinnen und Mitarbeiter aufgrund der primär quantitativen und im Kern betriebswirtschaftlichen Ausrichtung. Es trat die Notwendigkeit einer qualitativen Erweiterung der Perspektive zutage.

Dieser Erkenntnis folgend, wurde der Prozess der Implementierung durch eine Evaluationsstudie im Rahmen einer Diplomarbeit an der Fachhochschule Krems untersucht. Dabei wurde ein Compliance-Gefälle zwischen Führungs- und Mitarbeitendenebene identifiziert, das Kommunikations- und Beteiligungspotenziale in der Weiterentwicklung und Implementierung des Instrumentes bei den Beschäftigten erkennen ließ.

Diesen Ergebnissen folgend wurde vom Management die Entscheidung getroffen, das Instrument der Balanced Score Card um qualitative Aspekte der Wirkungsorientierung zu erweitern und damit den Nutzwert des Instrumentes als Orientierungs- und Reflexionsbasis für die operative Arbeit zu steigern. Dies sollte unter Beteiligung der Mitarbeiterschaft erfolgen.

Dazu wurde eine Steuerungsgruppe aus leitenden Mitarbeitenden und der Geschäftsführung gebildet, die einen Entwurf der qualitativ erweiterten Balanced Score Card vorlegen sollte, der in einer Groß- gruppenklausur mit allen Beschäftigten reflektiert wurde. Die Ergebnisse dieser Klausur wurden in den Steuergruppenentwurf eingearbeitet und das so adaptierte Modell als Grundlage für die Erarbeitung bereichsspezifischer Maßnahmenkataloge herangezogen. Damit sollen die Maßnahmen anhand der definierten organisationalen Ziele, Messgrößen und Zielwerte der Balanced Score Card auf die konkreten operativen Gegebenheiten der unterschiedlichen Arbeitsbereiche des Trägers heruntergebrochen werden.

Die Ergebnisse dieses Prozesses werden im Herbst 2012 in die bereichsinternen Entscheidungsphasen einmünden, deren Startup eine Leiterklausur darstellt, in der die Ergebnisse des Maßnahmenplanungsprozesses eingebracht werden. Geplant ist im Weiteren ein Prozess aus Rückkoppelungsschleifen zwischen den Leitungsebenen einerseits und der Leitungs- und Mitarbeitendenebene andererseits. Am Ende dieses Prozesses soll das organisationale Lernen und die institutionelle Weiterentwicklung des Trägers eine für alle Stakeholder anschlussfähige methodische Basis finden, die das gesamte Interessensspektrum aus Legitimation und organisationalem Lernen abzudecken vermag.

\section{Daniela Neubert}

Daniela Neubert hat Erziehungs- und Bildungswissenschaften und Sozialmanagement an den Universitäten Graz und Linz studiert. Sie war in Einrichtungen der Jugend- und Suchthilfe u. a. als Projektleiterin tätig und hatte eine Professur für Soziale Arbeit des Alters an der Fachhochschule Kärnten. Im Jahre 2010 trat sie "pro mente kärnten « bei und ist seither dort als Regionalmanagerin und als Leiterin des Sozialpsychiatrischen Zentrums Spittal/Drau tätig.

daniela.neubert@promente-kaernten.at
Lernende Organisationen setzen auf Vertrauen - was mit einem positiven Menschenbild und einer wertschätzenden Fehlerkultur Hand in Hand geht (vgl. Abb. 2).

Wie in jedem Veränderungsprozess, so ist auch bei der Entwicklung und Implementierung eines wirkungsorientieren Steuerungssystems zunächst einmal mit Angst und Widerstand zu rechnen. Auf wirkungsorientierte Steu- erung übertragen bedeutet dies, als erstes Verständnis für die Hintergründe zu schaffen und die Motivation der betroffenen Mitarbeitenden zu wecken. Danach werden bisheriger Ziele und Vorgehensweisen reflektiert, um die Bereitschaft zu schaffen, neue Wege zu denken. In einem dritten und letzten Schritt sollen die neuen Modelle verinnerlicht und zu einer Selbstverständlichkeit werden.

\section{Ausblick}

Im Zuge der sich verschärfenden Diskussion um den Einsatz öffentlicher Ressourcen und der damit verbundenen Kürzungen wird Wirkungsorientierung häufig als Vorwand eingesetzt, um Mittel zu kürzen - ohne jeglichen inhaltlichen Diskurs.

Das entspricht nicht dem eigentlichen Gedanken der Wirkungsorientierung, 
der sich primär nicht mir der Höhe der eingesetzten Mittel, sondern mit deren Angemessenheit im Hinblick auf die erreichten Veränderungen auseinandersetzt. Wenn jedoch Sparmaßnahmen im Vordergrund stehen und vorrangig Kontrolle anstatt Controlling eingefordert wird, ist die Gefahr groß, dass Wirkungsorientierung zu Legitimierungszwecken degradiert wird. Dann geht die Möglichkeit, aus dem Prozess Lerneffekte für die Organisation zu erzielen, verloren.

Ihr größtes Potenzial entfaltet wirkungsorientierte Steuerung, wenn sie zunächst einmal losgelöst von finanziellen Aspekten betrachtet wird. Eine Organisation, die über ihre Wirkungen reflektiert, schafft die Basis für einen nachhaltigen Lernprozess, der jedenfalls Früchte bringt - unabhängig von den Stakeholdern und dem damit verbundenen Hinterfragen der Legitimation.

Gerade ohne äußeren Druck, sondern aus einer intrinsischen Überzeugung der Führungsebene, lassen sich Wirkungsketten und Wirkungskennzahlen in einem Klima der Offenheit und Lernbereitschaft am besten erarbeiten.

\section{Literatur}

Bono, M. L. (2010): Performance Management in NPOs. Steuerung im Dienste sozialer Ziele, Baden-Baden.

Böhm, S. (2008): Organisationale Identifikation als Voraussetzung für eine erfolgreiche Unternehmensentwicklung, Wiesbaden. Heidenbrink, Markus/Jenewein, Wolfgang (2011): High-Performance-Organisationen, Stuttgart.

Pleier, N. (2008): Performance-MeasurementSysteme und der Faktor Mensch, Wiesbaden. Bono, M. L./Neubert, D. (2012): Wirkungsorientiertes Controlling im Spannungsverhältnis zwischen Legitimation und organisationalem Lernen. In: Strunk, A. (Hg.): Leitbildentwicklung und systemisches Controlling, Baden-Baden.

\section{SGB III-Reform bereits berücksichtigt}

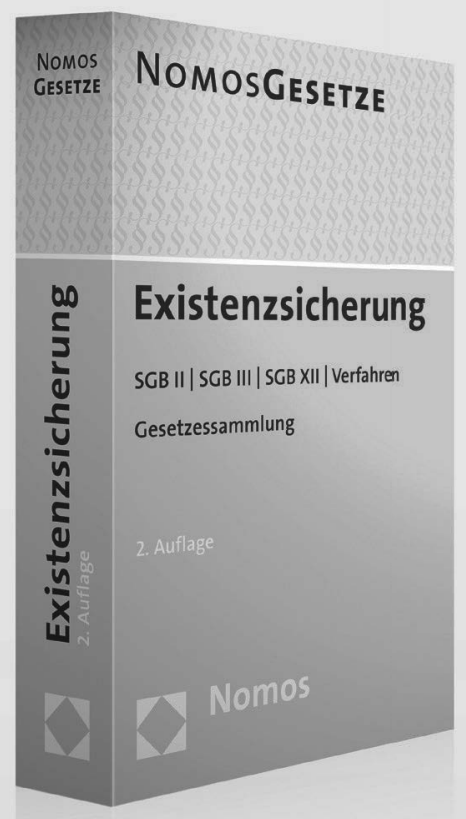

Mit dem Gesetz zur Verbesserung der Eingliederungschancen am Arbeitsmarkt vom 27.12.2011 sind einschneidende Reformen der arbeitsmarktpolitischen Eingliederungsinstrumente beschlossen. Rechtsansprüche sind verändert, Maßnahmen sind gestrichen und das SGB III ist umfassend geändert.

Die Textsammlung berücksichtigt diese umfangreichen Änderungen. Sie bringt neben dem ebenso betroffenen SGB II auch alle anderen (Sozial-) Gesetze, die einen notwendigen Bezug zum Existenzsicherungsrecht/Recht der Arbeitsvermittlung aufweisen, auf den allerneuesten Stand. Dies gilt genauso für die relevanten verfahrensrechtlichen Gesetze, die u.a. durch das Gesetz über den Rechtsschutz bei überlangen Gerichtsverfahren Änderungen unterlagen.

Die aktuelle und handliche Ausgabe ist für Berater wie für Betroffene gleichermaßen geeignet.

\section{Weitere Informationen: www.nomos-shop.de/14543}

\section{Existenzsicherung \\ SGB II | SGB III | SGB XII | \\ Verfahren \\ Gesetzessammlung \\ 2. Auflage 2012, 799 S., brosch., \\ 9,90€, ISBN 978-3-8329-7346-9

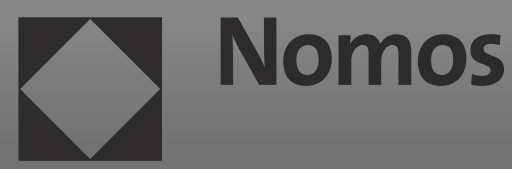


"Man kann erst steuern, wenn man Fahrt hat."

Emil Gött, deutscher Schriftsteller (1864-1908)

„Wenn man das Steuer in der Hand hat, spricht man davon, dass man gemeinsam in einem Boot sitzt." Gerhard Uhlenbruck, deutscher Arzt und Schriftsteller (geb. 1929)

"Alle, die im Stau stecken, sind schuld am Stau."

Rolf Dobelli, Schweizer Unternehmer und Schriftsteller (geb. 1966)

"Gibt es für ein Problem keine Lösung, so ändern wir das Problem! « Wolfgang Topp, Ex-Geschäftsführer der Märklin \& Cie GmbH

"Das unfehlbare Mittel, Autorität über Menschen zu

gewinnen, ist, sich ihnen nützlich zu machen."

Marie von Ebner-Eschenbach, österreichische Schriftstellerin (1830-1916)

"Manche freilich müssen drunten sterben, wo die schweren Ruder der Schiffe streifen, andere wohnen bei dem Steuer droben, kennen Vogelfilug und die Länder der Sterne. « Hugo von Hofmannsthal, österreichischer Schriftsteller (1874-1929)

"Ohne Wirtschaftlichkeit werden wir es nicht durchhalten und ohne Menschlichkeit werden wir es nicht aushalten." Basina Kloos, deutsche Ordensschwester und Geschäftsführerin der Marienhaus Gesundheits- und Sozialholding GmbH 